\title{
The ecology of medical care for adults in Alberta, 2002/03 to 2016/17: a retrospective cohort study
}

\author{
Finlay A. McAlister MD MSc, Marcello Tonelli MD MPH, Natasha Wiebe MMath PStat, Meng Lin MSc, \\ Lawrence W. Svenson $\mathrm{PhD}$, Stafford Dean $\mathrm{PhD}$
}

\section{Abstract}

Background: If we are to improve the patient experience, knowing where and with whom people receive professional health advice and treatment (the ecology of medical care) is the first step. We designed this study to define the ecology of medical care in Alberta and to examine whether province-wide implementation of 5 policy changes between 2003 and 2012 changed patterns of care among adults in the province.

Methods: This was a retrospective cohort study of adults (age $\geq 18 \mathrm{yr}$ ) in Alberta using routinely collected data from 6 linked administrative health databases, the 2016 Canadian Community Health Survey and the Alberta Health Link teletriage system. We collected data on all encounters with pharmacists, primary care physicians, specialists, emergency departments and hospitals in 2002/03, $2009 / 10$ and $2016 / 17$.

Results: Between 2002/03 and 2016/17, the community-dwelling adult population of Alberta increased from 2.66 million to 3.84 million; the median age increased from 41 to 43 years, and the proportion with at least 1 ambulatory-care-sensitive condition increased from $20.6 \%$ to $27.8 \%$. The proportion who saw a primary care physician decreased significantly (from $70.8 \%$ to $68.2 \%, p<0.001$ ), as did the proportion who visited an emergency department (from $20.6 \%$ to $19.2 \%, p<0.001$ ); the declines were seen in all subgroups examined. The proportion who saw a specialist as an outpatient increased from $31.9 \%$ to $33.2 \%(p<0.001)$, and the proportion who received at least 1 medication dispensation increased from $54.9 \%$ to $60.2 \%(p<0.001)$. The proportion admitted to an acute care hospital $(5.6 \%-6.5 \%)$ or academic hospital (1.2\%) was relatively stable over time.

Interpretation: Despite implementation of 5 system-wide changes designed to affect the delivery of primary and specialty medical care as well as the use of pharmacist and nursing services in Alberta, patterns of health care delivery changed little between $2002 / 03$ and 2016/17. Rather than searching for a policy "magic bullet," health care planners may be better served by focusing on upscaling and implementing interventions proven to be efficacious.

health care system cannot be judged solely on the basis of morbidity and mortality rates. Increasing recognition of the importance of the patient experience has refocused attention on the ecology of medical care: if we are to improve patient experience, knowing where and with whom people receive professional health advice and treatment is the first step. Studies using UK, US and Australian data from the 1930s to the early 2000s showed that, although most adults are healthy most of the time, $75 \%-$ $80 \%$ report symptoms at least once a year; less than onequarter see a physician for those symptoms, and less than $1 \%$ are admitted to hospital in any given month. ${ }^{1-4}$ The ecology of medical care differs across settings and is influenced by patient age, sex, socioeconomic status, diagnoses and comorbidity profiles. ${ }^{5-7}$

We conducted this study to define the ecology of medical care in Alberta and to examine whether it changed after 5 pol- icy changes were implemented province-wide by the health ministry and Alberta Health Services between 2003 and 2012 (Box 1). ${ }^{8-11}$ We also explored the care received by subgroups defined by demographic characteristics, area of residence and whether patients had ambulatory-care-sensitive conditions (ACSCs), defined by the Canadian Institute of Health Information as asthma, chronic obstructive pulmonary disease, heart failure, coronary disease, hypertension, diabetes or epilepsy. ${ }^{12}$

Competing interests: None declared.

This article has been peer reviewed.

Correspondence to: Finlay McAlister, Finlay.McAlister@ualberta.ca

CMAJ Open 2020. DOI:10.9778/cmajo.20190188 


\section{Box 1: Five system-wide policy changes implemented in} Alberta between 2003 and 2012

1. The Alberta Health Link teletriage system was established in the latter half of 2003 as a means to try to decant some care to nonphysician providers. ${ }^{11}$ The system is staffed by specially trained nurses using clinical decision support software to assist with symptom triage of callers and to select 1 of 18 recommendations about whether to seek inperson evaluation by medical, pharmacist or home care staff, and how promptly that should be done.

2. Primary Care Networks were established in 2005 to help facilitate access to primary care and implement chronic disease management programs. ${ }^{8}$ Primary Care Networks include primary care physicians (reimbursed on a fee-forservice basis) and nonphysician health care providers (such as nurses, dietitians and pharmacists) funded per capita to provide team-based preventive health or chronic disease management for a roster of patients.

3. The remuneration model for most academic specialists was converted from fee-for-service to salary between 2005 and 2007. ${ }^{9}$

4. Specialty medicine was organized into province-wide Strategic Clinical Networks starting in 2012 (there were initially 5, but as of 2019 there were 16). Strategic Clinical Networks are partnerships between clinicians and consumers organized by disease area that identify priorities, explore solutions and implement strategies to improve care and outcomes. ${ }^{10}$

5. As of 2012, pharmacists were allowed to bill for providing comprehensive annual care plans and ongoing disease management to patients with selected chronic diseases or for modifying or prescribing medications as the prescriber of record (www.ab.bluecross.ca/pdfs/pharmacy-benefacts/346 -compensation-for-pharmacy-services.pdf).

\section{Methods}

\section{Design and setting}

This was a retrospective cohort study of adults in Alberta using routinely collected data from 6 linked administrative health databases to determine health care use per annum from Apr. 1, 2002 to Mar. 31, 2003, from Apr. 1, 2009 to Mar. 31, 2010, and from Apr. 1, 2016 to Mar. 31, 2017. We also used the 2016 edition of the Canadian Community Health Survey $^{13}$ (a random survey of community-dwelling adults that is anonymous and thus not linked to their health records) and the Alberta Health Link teletriage database for 2018 (prior years did not have complete data capture). We chose 2002/03 and 2016/17 to compare the ecology of care before and after implementation of the 5 policy changes.

The study was set in the Canadian province of Alberta, which has a government-funded health care system that provides universal access to hospital, emergency department and physician services. Services are free to the patient at the point of care.

\section{Data sources and study sample}

All adults aged 18 or more resident in Alberta during study periods were included in the study sample, regardless of whether they were community-dwelling or in long-term care.
We excluded patients without Alberta Health Care Insurance Plan coverage (for example, people from other jurisdictions receiving care in Alberta facilities).

The administrative databases we used included the Discharge Abstract Database, the Ambulatory Care Database (which records dates for all emergency department visits), the Alberta HealthCare Provider Claims Database (which captures dates for all physician claims, including those shadow-billed by salaried physicians), the Alberta Pharmaceutical Information Network (which captures dates for all medication dispensations from community pharmacies in the province after 2008) and the Alberta Health Care Insurance Plan Registry (which includes patient postal code, permitting comparisons between residents of rural v. urban areas). We used patient health insurance numbers to link all data sets. We interrogated the Alberta Blue Cross Pharmacy Services database at Alberta Health, which captures publicly funded pharmacy consultative services (not visits for medication dispensations but, rather, pharmacy visits during which the pharmacist modifies or prescribes medications, or delivers patient counselling in areas such as smoking cessation or chronic disease management). The accuracy and comprehensiveness of the Alberta administrative databases we used in this study have been previously established. ${ }^{14,15}$

We used the 2016 Canadian Community Health Survey (most recent year available) to determine the proportion of community-dwelling adults in Alberta who reported their health as excellent or very good, and the proportion who reported that they had unmet health care needs.

Alberta Health Link is a provincial teletriage service that receives more than 1 million calls annually (https://www. albertahealthservices.ca/assets/healthinfo/link/index.html). As a person may call Health Link on behalf of somebody else (usually a parent calling about an illness in his or her child), we defined the caller as the person with the health problem prompting the call.

\section{Comorbidities}

We identified comorbidities for each patient using International Classification of Diseases, 9th Revision and International Statistical Classification of Diseases and Related Health Problems, 10th Revision codes for any hospital admissions, emergency department visits and outpatient visits in the databases before and including the index visit using case definitions previously validated in Alberta. ${ }^{14}$ Case definitions for ACSCs are provided in Appendix 1 (available at www.cmajopen.ca/content/ 8/1/E169/suppl/DC1).

\section{Outcomes}

Our primary outcomes were the proportion of adults who had encounters with pharmacists, primary care physicians, specialists, emergency departments or hospitals in 2002/03, 2009/10 and 2016/17, and the proportion who accessed Alberta Health Link teletriage resources.

\section{Statistical analysis}

We report the proportion of adults who received each service on a yearly basis in the 3 years studied, and compared between 
subgroups defined by patient demographic characteristics (sex, age strata), geographic area (rural v. urban) and presence or absence of ACSCs. We report the frequencies for longterm care residents separately from those for communitydwelling people. We assessed differences for statistical significance $(p<0.05)$ using the $\chi^{2}$ test and tested for trends across years using a nonparametric test by Cuzick. ${ }^{16}$

\section{Ethics approval}

The University of Alberta Health Research Ethics Board approved this study and granted a waiver for individual patient consent as the investigators were provided only with deidentified data.

\section{Results}

Between 2002/03 and 2016/17, the population of communitydwelling adults in Alberta increased from 2.66 million to 3.84 million, and the number of long-term care residents increased from 25853 to 44 199; the sex, rural versus urban and long-term care distributions did not change appreciably over the study period (Tables 1-3). However, the median age increased from 41 to 43 years, and the proportion of adults with at least 1 ACSC increased from $20.6 \%$ to $27.8 \%$.

\section{Physician services}

The proportion of community-dwelling adults who saw a primary care physician at least once a year decreased from $70.8 \%$ to $68.2 \%$ over the study period, and the proportion who visited an emergency department declined from $20.6 \%$ to $19.2 \%$ (both $p<0.001$ ) (Figure 1). The declines were seen in all subgroups of community-dwelling people examined, including older people and those with ACSCs. The proportion of community-dwelling people who saw a specialist as an outpatient increased over time, from $31.9 \%$ to $33.2 \%$ ( $p<$ $0.001)$ for any specialist and from $2.2 \%$ to $4.5 \%(p<0.001)$ for salaried specialists. However, the proportions of adults admitted to hospital or admitted to an academic hospital were relatively stable between 2002/03 and 2016/17 (about 6\% and $1 \%$, respectively, in community-dwelling people, and $35 \%$ and $10 \%$ in long-term care residents).

\section{Pharmacist services}

Pharmacy dispensation data were not collected in 2003. The proportion of people who received at least 1 medication dispensation from a community pharmacy per year increased between $2009 / 10$ and $2016 / 17$, from $54.9 \%$ to $60.2 \%$ ( $p<$ 0.001 ) in community-dwelling adults and from $70.3 \%$ to $82.9 \%(p<0.001)$ in long-term care residents; the increases

Table 1: Use of health care services by adults in Alberta, 2002/03

\begin{tabular}{|c|c|c|c|c|c|c|c|c|c|c|c|}
\hline \multirow[b]{3}{*}{ Variable } & \multicolumn{10}{|c|}{ Community-dwelling, no. (\%)* } & \multirow{3}{*}{$\begin{array}{c}\text { Long-term } \\
\text { care } \\
\text { residents, } \\
\text { no. }(\%)^{*} \\
n= \\
25853\end{array}$} \\
\hline & \multirow[b]{2}{*}{$\begin{array}{c}\text { Overall } \\
n= \\
2660947\end{array}$} & \multicolumn{2}{|c|}{ Residence } & \multicolumn{3}{|c|}{ Age, yr } & \multicolumn{2}{|c|}{ Sex } & \multicolumn{2}{|c|}{ No. of ACSCs } & \\
\hline & & $\begin{array}{c}\text { Urban } \\
n= \\
2206514\end{array}$ & $\begin{array}{c}\text { Rural } \\
n= \\
295685\end{array}$ & $\begin{array}{c}18-40 \\
n= \\
1251465\end{array}$ & $\begin{array}{c}41-65 \\
n= \\
1086555\end{array}$ & $\begin{array}{c}>65 \\
n= \\
322927\end{array}$ & $\begin{array}{c}\text { Male } \\
n= \\
1353551\end{array}$ & $\begin{array}{c}\text { Female } \\
n= \\
1307396\end{array}$ & $\begin{array}{c}0 \\
n= \\
2113374\end{array}$ & $\begin{array}{c}\geq 1 \\
n= \\
547573\end{array}$ & \\
\hline Age, yr, median (IQR) & $\begin{array}{c}41 \\
(29-53)\end{array}$ & $\begin{array}{c}41 \\
(29-53)\end{array}$ & $\begin{array}{c}43 \\
(31-56)\end{array}$ & $\begin{array}{c}28 \\
(23-34)\end{array}$ & $\begin{array}{c}49 \\
(44-55)\end{array}$ & $\begin{array}{c}73 \\
(69-79)\end{array}$ & $\begin{array}{c}40 \\
(29-52)\end{array}$ & $\begin{array}{c}41 \\
(29-54)\end{array}$ & $\begin{array}{c}37 \\
(27-47)\end{array}$ & $\begin{array}{c}60 \\
(48-71)\end{array}$ & $\begin{array}{c}82 \\
(73-88)\end{array}$ \\
\hline Female sex & $\begin{array}{c}1307396 \\
\quad(49.1)\end{array}$ & $\begin{array}{c}1099863 \\
(49.8)\end{array}$ & $\begin{array}{c}142766 \\
(48.3)\end{array}$ & $\begin{array}{l}601486 \\
(48.1)\end{array}$ & $\begin{array}{c}529133 \\
(48.7)\end{array}$ & $\begin{array}{c}176777 \\
(54.7)\end{array}$ & $\begin{array}{c}0 \\
(0.0)\end{array}$ & $\begin{array}{c}1307396 \\
(100.0)\end{array}$ & $\begin{array}{l}1020754 \\
(48.3)\end{array}$ & $\begin{array}{c}286642 \\
(52.3)\end{array}$ & $\begin{array}{l}16743 \\
(64.8)\end{array}$ \\
\hline $\begin{array}{l}\text { No. of comorbidities, } \\
\text { median (IQR) }\end{array}$ & $\begin{array}{c}0 \\
(0-1)\end{array}$ & $\begin{array}{c}0 \\
(0-1)\end{array}$ & $\begin{array}{c}1 \\
(0-1)\end{array}$ & $\begin{array}{c}0 \\
(0-1)\end{array}$ & $\begin{array}{c}1 \\
(0-1)\end{array}$ & $\begin{array}{c}2 \\
(1-3)\end{array}$ & $\begin{array}{c}0 \\
(0-1)\end{array}$ & $\begin{array}{c}0 \\
(0-1)\end{array}$ & $\begin{array}{c}0 \\
(0-1)\end{array}$ & $\begin{array}{c}2 \\
(1-3)\end{array}$ & $\begin{array}{c}4 \\
(3-6)\end{array}$ \\
\hline Urban residence $†$ & $\begin{array}{c}2206514 \\
(88.2)\end{array}$ & $\begin{array}{c}2206514 \\
(100.0)\end{array}$ & $\begin{array}{c}0 \\
(0.0)\end{array}$ & $\begin{array}{c}1024656 \\
(89.1)\end{array}$ & $\begin{array}{l}911686 \\
(87.6)\end{array}$ & $\begin{array}{c}270172 \\
(87.0)\end{array}$ & $\begin{array}{c}1106651 \\
(87.9)\end{array}$ & $\begin{array}{c}1099863 \\
(88.5)\end{array}$ & $\begin{array}{c}1736468 \\
(88.6)\end{array}$ & $\begin{array}{c}470046 \\
(86.8)\end{array}$ & $\begin{array}{c}23338 \\
(90.9)\end{array}$ \\
\hline $\begin{array}{l}\text { Saw primary care } \\
\text { physician in office } \\
\text { setting }\end{array}$ & $\begin{array}{c}1883353 \\
(70.8)\end{array}$ & $\begin{array}{c}1661432 \\
(75.3)\end{array}$ & $\begin{array}{c}215357 \\
(72.8)\end{array}$ & $\begin{array}{l}796915 \\
(63.7)\end{array}$ & $\begin{array}{c}807792 \\
(74.3)\end{array}$ & $\begin{array}{c}278646 \\
(86.3)\end{array}$ & $\begin{array}{l}833798 \\
(61.6)\end{array}$ & $\begin{array}{c}1049555 \\
(80.3)\end{array}$ & $\begin{array}{c}1377949 \\
(65.2)\end{array}$ & $\begin{array}{c}505404 \\
(92.3)\end{array}$ & $\begin{array}{l}11452 \\
(44.3)\end{array}$ \\
\hline $\begin{array}{l}\text { Saw salaried specialist } \\
\text { as outpatient }\end{array}$ & $\begin{array}{l}58979 \\
(2.2)\end{array}$ & $\begin{array}{l}52898 \\
(2.4)\end{array}$ & $\begin{array}{l}5914 \\
(2.0)\end{array}$ & $\begin{array}{c}15459 \\
(1.2)\end{array}$ & $\begin{array}{c}26622 \\
(2.5)\end{array}$ & $\begin{array}{c}16898 \\
(5.2)\end{array}$ & $\begin{array}{c}26204 \\
(1.9)\end{array}$ & $\begin{array}{c}32775 \\
(2.5)\end{array}$ & $\begin{array}{c}29217 \\
(1.4)\end{array}$ & $\begin{array}{l}29762 \\
(5.4)\end{array}$ & $\begin{array}{l}1069 \\
(4.1)\end{array}$ \\
\hline $\begin{array}{l}\text { Saw FFS specialist as } \\
\text { outpatient }\end{array}$ & $\begin{array}{c}838916 \\
(31.5)\end{array}$ & $\begin{array}{c}759658 \\
(34.4)\end{array}$ & $\begin{array}{l}77230 \\
(26.1)\end{array}$ & $\begin{array}{c}268626 \\
(21.5)\end{array}$ & $\begin{array}{c}384902 \\
(35.4)\end{array}$ & $\begin{array}{l}185388 \\
(57.4)\end{array}$ & $\begin{array}{c}344465 \\
(25.4)\end{array}$ & $\begin{array}{l}494451 \\
(37.8)\end{array}$ & $\begin{array}{l}529599 \\
(25.1)\end{array}$ & $\begin{array}{c}309317 \\
(56.5)\end{array}$ & $\begin{array}{c}10780 \\
(41.7)\end{array}$ \\
\hline $\begin{array}{l}\text { Saw any specialist as } \\
\text { outpatient }\end{array}$ & $\begin{array}{c}849668 \\
(31.9)\end{array}$ & $\begin{array}{l}768955 \\
(34.8)\end{array}$ & $\begin{array}{l}78584 \\
(26.6)\end{array}$ & $\begin{array}{c}272436 \\
(21.8)\end{array}$ & $\begin{array}{c}389988 \\
(35.9)\end{array}$ & $\begin{array}{c}187244 \\
(58.0)\end{array}$ & $\begin{array}{l}350055 \\
(25.9)\end{array}$ & $\begin{array}{c}499613 \\
(38.2)\end{array}$ & $\begin{array}{c}536064 \\
(25.4)\end{array}$ & $\begin{array}{c}313604 \\
(57.3)\end{array}$ & $\begin{array}{l}10917 \\
(42.2)\end{array}$ \\
\hline $\begin{array}{l}\text { Had at least } \\
1 \text { emergency } \\
\text { department visit }\end{array}$ & $\begin{array}{c}549039 \\
(20.6)\end{array}$ & $\begin{array}{l}450325 \\
(20.4)\end{array}$ & $\begin{array}{c}97354 \\
(32.9)\end{array}$ & $\begin{array}{c}259600 \\
(20.7)\end{array}$ & $\begin{array}{l}199755 \\
(18.4)\end{array}$ & $\begin{array}{c}89684 \\
(27.8)\end{array}$ & $\begin{array}{l}273085 \\
(20.2)\end{array}$ & $\begin{array}{c}275954 \\
(21.1)\end{array}$ & $\begin{array}{c}375784 \\
(17.8)\end{array}$ & $\begin{array}{c}173255 \\
(31.6)\end{array}$ & $\begin{array}{l}11586 \\
(44.8)\end{array}$ \\
\hline $\begin{array}{l}\text { Had at least } 1 \text { hospital } \\
\text { admission } \ddagger\end{array}$ & $\begin{array}{c}173179 \\
(6.5)\end{array}$ & $\begin{array}{c}144560 \\
(6.6)\end{array}$ & $\begin{array}{c}28436 \\
(9.6)\end{array}$ & $\begin{array}{c}70102 \\
(5.6)\end{array}$ & $\begin{array}{l}54580 \\
(5.0)\end{array}$ & $\begin{array}{l}48497 \\
(15.0)\end{array}$ & $\begin{array}{c}64116 \\
(4.7)\end{array}$ & $\begin{array}{c}109063 \\
(8.3)\end{array}$ & $\begin{array}{l}92465 \\
(4.4)\end{array}$ & $\begin{array}{l}80714 \\
(14.7)\end{array}$ & $\begin{array}{l}9127 \\
(35.3)\end{array}$ \\
\hline
\end{tabular}


Table 2: Use of health care services by adults in Alberta, 2009/10

\begin{tabular}{|c|c|c|c|c|c|c|c|c|c|c|c|}
\hline \multirow[b]{3}{*}{ Variable } & \multicolumn{10}{|c|}{ Community-dwelling, no. $(\%)^{*}$} & \multirow{3}{*}{$\begin{array}{c}\text { Long-term } \\
\text { care } \\
\text { residents, } \\
\text { no. }(\%)^{\star} \\
n= \\
34121\end{array}$} \\
\hline & \multirow[b]{2}{*}{$\begin{array}{c}\text { Overall } \\
n= \\
3261459\end{array}$} & \multicolumn{2}{|c|}{ Residence } & \multicolumn{3}{|c|}{ Age, yr } & \multicolumn{2}{|c|}{ Sex } & \multicolumn{2}{|c|}{ No. of ACSCs } & \\
\hline & & $\begin{array}{c}\text { Urban } \\
n= \\
2731980\end{array}$ & $\begin{array}{c}\text { Rural } \\
n= \\
339996\end{array}$ & $\begin{array}{c}18-40 \\
n= \\
1492764\end{array}$ & $\begin{array}{c}41-65 \\
n= \\
1369231\end{array}$ & $\begin{array}{c}>65 \\
n= \\
399464\end{array}$ & $\begin{array}{c}\text { Male } \\
n= \\
1672187\end{array}$ & $\begin{array}{c}\text { Female } \\
n= \\
1589272\end{array}$ & $\begin{array}{c}0 \\
n= \\
2458843\end{array}$ & $\begin{array}{c}\geq 1 \\
n= \\
802616\end{array}$ & \\
\hline Age, yr, median (IQR) & $\begin{array}{c}42 \\
(29-55)\end{array}$ & $\begin{array}{c}42 \\
(29-55)\end{array}$ & $\begin{array}{c}44 \\
(29-57)\end{array}$ & $\begin{array}{c}28 \\
(23-34)\end{array}$ & $\begin{array}{c}50 \\
(45-56)\end{array}$ & $\begin{array}{c}73 \\
(68-79)\end{array}$ & $\begin{array}{c}41 \\
(29-54)\end{array}$ & $\begin{array}{c}42 \\
(29-55)\end{array}$ & $\begin{array}{c}37 \\
(27-48)\end{array}$ & $\begin{array}{c}59 \\
(49-70)\end{array}$ & $\begin{array}{c}82 \\
(71-88)\end{array}$ \\
\hline Female sex & $\begin{array}{c}1589272 \\
(48.7)\end{array}$ & $\begin{array}{c}1346267 \\
(49.3)\end{array}$ & $\begin{array}{c}165347 \\
(48.6)\end{array}$ & $\begin{array}{c}715257 \\
(47.9)\end{array}$ & $\begin{array}{c}660282 \\
(48.2)\end{array}$ & $\begin{array}{c}213733 \\
(53.5)\end{array}$ & $\begin{array}{c}0 \\
(0.0)\end{array}$ & $\begin{array}{c}1589272 \\
(100.0)\end{array}$ & $\begin{array}{c}1182963 \\
(48.1)\end{array}$ & $\begin{array}{c}406309 \\
(50.6)\end{array}$ & $\begin{array}{l}21164 \\
(62.0)\end{array}$ \\
\hline $\begin{array}{l}\text { No. of comorbidities, } \\
\text { median (IQR) }\end{array}$ & $\begin{array}{c}0 \\
(0-1)\end{array}$ & $\begin{array}{c}0 \\
(0-2)\end{array}$ & $\begin{array}{c}1 \\
(0-2)\end{array}$ & $\begin{array}{c}0 \\
(0-1)\end{array}$ & $\begin{array}{c}1 \\
(0-2)\end{array}$ & $\begin{array}{c}3 \\
(1-4)\end{array}$ & $\begin{array}{c}0 \\
(0-1)\end{array}$ & $\begin{array}{c}1 \\
(0-2)\end{array}$ & $\begin{array}{c}0 \\
(0-1)\end{array}$ & $\begin{array}{c}2 \\
(2-4)\end{array}$ & $\begin{array}{c}6 \\
(4-7)\end{array}$ \\
\hline Urban residence† & $\begin{array}{c}2731980 \\
(88.9)\end{array}$ & $\begin{array}{c}2731980 \\
(100.0)\end{array}$ & $\begin{array}{c}0 \\
(0.0)\end{array}$ & $\begin{array}{c}1246856 \\
(89.7)\end{array}$ & $\begin{array}{c}1154496 \\
(88.8)\end{array}$ & $\begin{array}{c}330628 \\
(86.5)\end{array}$ & $\begin{array}{c}1385713 \\
(88.8)\end{array}$ & $\begin{array}{c}1346267 \\
(89.1)\end{array}$ & $\begin{array}{c}2036905 \\
(89.5)\end{array}$ & $\begin{array}{c}695075 \\
(87.4)\end{array}$ & $\begin{array}{l}30109 \\
(88.8)\end{array}$ \\
\hline $\begin{array}{l}\text { Saw primary care } \\
\text { physician in office } \\
\text { setting }\end{array}$ & $\begin{array}{c}2213242 \\
\quad(67.9)\end{array}$ & $\begin{array}{c}1966992 \\
(72.0)\end{array}$ & $\begin{array}{c}242026 \\
(71.2)\end{array}$ & $\begin{array}{c}888408 \\
(59.5)\end{array}$ & $\begin{array}{c}985739 \\
(72.0)\end{array}$ & $\begin{array}{c}339095 \\
(84.9)\end{array}$ & $\begin{array}{c}985842 \\
(59.0)\end{array}$ & $\begin{array}{c}1227400 \\
(77.2)\end{array}$ & $\begin{array}{c}1486193 \\
\quad(60.4)\end{array}$ & $\begin{array}{c}727049 \\
(90.6)\end{array}$ & $\begin{array}{l}26655 \\
(78.1)\end{array}$ \\
\hline $\begin{array}{l}\text { Saw salaried } \\
\text { specialist as } \\
\text { outpatient }\end{array}$ & $\begin{array}{c}34357 \\
(1.1)\end{array}$ & $\begin{array}{c}30910 \\
(1.1)\end{array}$ & $\begin{array}{l}3337 \\
(1.0)\end{array}$ & $\begin{array}{l}8639 \\
(0.6)\end{array}$ & $\begin{array}{c}15261 \\
(1.1)\end{array}$ & $\begin{array}{c}10457 \\
(2.6)\end{array}$ & $\begin{array}{c}14521 \\
(0.9)\end{array}$ & $\begin{array}{c}19836 \\
(1.2)\end{array}$ & $\begin{array}{c}16766 \\
(0.7)\end{array}$ & $\begin{array}{c}17591 \\
(2.2)\end{array}$ & $\begin{array}{l}705 \\
(2.1)\end{array}$ \\
\hline $\begin{array}{l}\text { Saw FFS specialist as } \\
\text { outpatient }\end{array}$ & $\begin{array}{c}998159 \\
(30.6)\end{array}$ & $\begin{array}{c}902039 \\
(33.0)\end{array}$ & $\begin{array}{l}94234 \\
(27.7)\end{array}$ & $\begin{array}{c}301515 \\
(20.2)\end{array}$ & $\begin{array}{l}470601 \\
(34.4)\end{array}$ & $\begin{array}{c}226043 \\
(56.6)\end{array}$ & $\begin{array}{l}419346 \\
(25.1)\end{array}$ & $\begin{array}{c}578813 \\
(36.4)\end{array}$ & $\begin{array}{l}560979 \\
(22.8)\end{array}$ & $\begin{array}{c}437180 \\
(54.5)\end{array}$ & $\begin{array}{l}15796 \\
(46.3)\end{array}$ \\
\hline $\begin{array}{l}\text { Saw any specialist as } \\
\text { outpatient }\end{array}$ & $\begin{array}{c}999737 \\
(30.7)\end{array}$ & $\begin{array}{c}903392 \\
(33.1)\end{array}$ & $\begin{array}{c}94421 \\
(27.8)\end{array}$ & $\begin{array}{c}302290 \\
(20.3)\end{array}$ & $\begin{array}{l}471294 \\
(34.4)\end{array}$ & $\begin{array}{c}226153 \\
(56.6)\end{array}$ & $\begin{array}{c}420266 \\
(25.1)\end{array}$ & $\begin{array}{c}579471 \\
(36.5)\end{array}$ & $\begin{array}{c}562203 \\
(22.9)\end{array}$ & $\begin{array}{c}437534 \\
(54.5)\end{array}$ & $\begin{array}{l}15808 \\
(46.3)\end{array}$ \\
\hline $\begin{array}{l}\text { Had at least } \\
1 \text { medication } \\
\text { dispensation }\end{array}$ & $\begin{array}{c}1789513 \\
(54.9)\end{array}$ & $\begin{array}{c}1575859 \\
(57.7)\end{array}$ & $\begin{array}{c}212030 \\
(62.4)\end{array}$ & $\begin{array}{c}675570 \\
(45.3)\end{array}$ & $\begin{array}{l}797895 \\
(58.3)\end{array}$ & $\begin{array}{c}316048 \\
(79.1)\end{array}$ & $\begin{array}{l}769355 \\
(46.0)\end{array}$ & $\begin{array}{c}1020158 \\
(64.2)\end{array}$ & $\begin{array}{c}1123292 \\
\quad(45.7)\end{array}$ & $\begin{array}{c}666221 \\
(83.0)\end{array}$ & $\begin{array}{l}23983 \\
(70.3)\end{array}$ \\
\hline $\begin{array}{l}\text { Had at least } \\
1 \text { emergency } \\
\text { department visit }\end{array}$ & $\begin{array}{c}644247 \\
(19.8)\end{array}$ & $\begin{array}{c}526163 \\
(19.3)\end{array}$ & $\begin{array}{c}116673 \\
(34.3)\end{array}$ & $\begin{array}{c}295663 \\
(19.8)\end{array}$ & $\begin{array}{l}243185 \\
(17.8)\end{array}$ & $\begin{array}{c}105399 \\
(26.4)\end{array}$ & $\begin{array}{c}314769 \\
(18.8)\end{array}$ & $\begin{array}{c}329478 \\
(20.7)\end{array}$ & $\begin{array}{c}409589 \\
(16.7)\end{array}$ & $\begin{array}{c}234658 \\
(29.2)\end{array}$ & $\begin{array}{l}16268 \\
(47.7)\end{array}$ \\
\hline $\begin{array}{l}\text { Had at least } 1 \\
\text { hospital admission }\end{array}$ & $\begin{array}{c}192409 \\
(5.9)\end{array}$ & $\begin{array}{c}163362 \\
(6.0)\end{array}$ & $\begin{array}{c}28886 \\
(8.5)\end{array}$ & $\begin{array}{l}79556 \\
(5.3)\end{array}$ & $\begin{array}{c}61568 \\
(4.5)\end{array}$ & $\begin{array}{l}51285 \\
(12.8)\end{array}$ & $\begin{array}{c}69420 \\
(4.2)\end{array}$ & $\begin{array}{c}122989 \\
(7.7)\end{array}$ & $\begin{array}{c}98222 \\
(4.0)\end{array}$ & $\begin{array}{l}94187 \\
(11.7)\end{array}$ & $\begin{array}{l}12308 \\
(36.1)\end{array}$ \\
\hline $\begin{array}{l}\text { Admitted to academic } \\
\text { centre }\end{array}$ & $\begin{array}{c}40686 \\
(1.2)\end{array}$ & $\begin{array}{c}37000 \\
(1.4)\end{array}$ & $\begin{array}{l}3650 \\
(1.1)\end{array}$ & $\begin{array}{c}12251 \\
(0.8)\end{array}$ & $\begin{array}{c}15742 \\
(1.1)\end{array}$ & $\begin{array}{c}12693 \\
(3.2)\end{array}$ & $\begin{array}{c}19200 \\
(1.1)\end{array}$ & $\begin{array}{c}21486 \\
(1.4)\end{array}$ & $\begin{array}{c}16855 \\
(0.7)\end{array}$ & $\begin{array}{c}23831 \\
(3.0)\end{array}$ & $\begin{array}{l}3289 \\
(9.6)\end{array}$ \\
\hline
\end{tabular}

were seen in all subgroups examined (Table 2, Table 3). However, very few people received counselling from pharmacists or had their prescriptions modified by a pharmacist as an outpatient despite the addition of a specific billing code for this service (Table 3).

\section{Patient subgroups}

The gradients between demographic subgroups were in the directions expected for all 3 years examined: older patients, women and long-term care residents were more likely to have seen a primary care physician or specialist, received medications, visited an emergency department, been admitted to hospital and been admitted to an academic hospital. Some of the gradients were large: in 2016/17, compared to community-dwelling people without an ACSC, those with at least 1 ACSC were more likely to have seen their primary care physician (87.8\% v. 60.7\%), had at least 1 medication dispensed $(86.8 \%$ v. $50.0 \%)$, been seen as outpatients by a specialist ( $55.4 \%$ v. $24.6 \%$ ), had at least 1 emergency department visit $(28.5 \%$ v. $15.6 \%)$ and been admitted to hospital at least once $(10.7 \%$ v. $3.7 \%$ ) (all $p<0.001)$; proportions were similar in prior years. Moreover, community-dwelling adults with at least 1 ACSC were more likely than those without ACSCs to receive care from both primary care physicians and specialists (both fee-for-service and salaried specialists) as outpatients $(p<0.001)$ (Figure 2).

\section{Self-reported health status}

Overall, $64.2 \%$ of community-dwelling adults who participated in the 2016 Canadian Community Health Survey reported that their health was very good or excellent (ranging from $70.3 \%$ of those aged $18-40 \mathrm{yr}$ to $48.9 \%$ of those $>65 \mathrm{yr}$ ) (Appendix 2, available at www.cmajopen.ca/ content/8/1/E169/suppl/DC1). Respondents without any ACSCs were more likely to report excellent or very good health than those with 1 or more ACSCs (72.3\% v. $42.9 \%)$. The average score for respondents on the EQ-5D Canadian utilities visual analogue scale for health-related quality of life 
Table 3: Use of health care services by adults in Alberta, 2016/17

\begin{tabular}{|c|c|c|c|c|c|c|c|c|c|c|c|}
\hline \multirow[b]{3}{*}{ Variable } & \multicolumn{10}{|c|}{ Community-dwelling, no. $(\%)^{\star}$} & \multirow{3}{*}{$\begin{array}{c}\text { Long-term } \\
\text { care } \\
\text { residents } \\
n= \\
44199\end{array}$} \\
\hline & \multirow[b]{2}{*}{$\begin{array}{c}\text { Overall } \\
n= \\
3840527\end{array}$} & \multicolumn{2}{|c|}{ Residence } & \multicolumn{3}{|c|}{ Age, yr } & \multicolumn{2}{|c|}{ Sex } & \multicolumn{2}{|c|}{ No. of ACSCs } & \\
\hline & & $\begin{array}{c}\text { Urban } \\
n= \\
3264235\end{array}$ & $\begin{array}{c}\text { Rural } \\
n= \\
377048\end{array}$ & $\begin{array}{c}18-40 \\
n= \\
1664530\end{array}$ & $\begin{array}{c}41-65 \\
n= \\
1617231\end{array}$ & $\begin{array}{c}>65 \\
n= \\
558766\end{array}$ & $\begin{array}{c}\text { Male } \\
n= \\
1961342\end{array}$ & $\begin{array}{c}\text { Female } \\
n= \\
1879185\end{array}$ & $\begin{array}{c}0 \\
n= \\
2774814\end{array}$ & $\begin{array}{c}\geq 1 \\
n= \\
1065713\end{array}$ & \\
\hline $\begin{array}{l}\text { Age, yr, median } \\
\text { (IQR) }\end{array}$ & $\begin{array}{c}43 \\
(31-57)\end{array}$ & $\begin{array}{c}43 \\
(31-57)\end{array}$ & $\begin{array}{c}45 \\
(30-60)\end{array}$ & $\begin{array}{c}30 \\
(24-34)\end{array}$ & $\begin{array}{c}51 \\
(45-57)\end{array}$ & $\begin{array}{c}72 \\
(68-79)\end{array}$ & $\begin{array}{c}43 \\
(31-57)\end{array}$ & $\begin{array}{c}43 \\
(31-58)\end{array}$ & $\begin{array}{c}37 \\
(28-49)\end{array}$ & $\begin{array}{c}60 \\
(49-70)\end{array}$ & $\begin{array}{c}79 \\
(62-88)\end{array}$ \\
\hline Female sex & $\begin{array}{c}1879185 \\
\quad(48.9)\end{array}$ & $\begin{array}{c}1611933 \\
(49.4)\end{array}$ & $\begin{array}{c}184876 \\
(49.0)\end{array}$ & $\begin{array}{c}812923 \\
(48.8)\end{array}$ & $\begin{array}{c}775091 \\
(47.9)\end{array}$ & $\begin{array}{c}291171 \\
(52.1)\end{array}$ & $\begin{array}{c}0 \\
(0.0)\end{array}$ & $\begin{array}{c}1879185 \\
(100.0)\end{array}$ & $\begin{array}{c}1355404 \\
\quad(48.8)\end{array}$ & $\begin{array}{c}523781 \\
(49.1)\end{array}$ & $\begin{array}{l}25357 \\
(57.4)\end{array}$ \\
\hline $\begin{array}{l}\text { No. of comorbidities, } \\
\text { median (IQR) }\end{array}$ & $\begin{array}{c}0 \\
(0-2)\end{array}$ & $\begin{array}{c}1 \\
(0-2)\end{array}$ & $\begin{array}{c}1 \\
(0-2)\end{array}$ & $\begin{array}{c}0 \\
(0-1)\end{array}$ & $\begin{array}{c}1 \\
(0-2)\end{array}$ & $\begin{array}{c}3 \\
(1-4)\end{array}$ & $\begin{array}{c}0 \\
(0-2)\end{array}$ & $\begin{array}{c}1 \\
(0-2)\end{array}$ & $\begin{array}{c}0 \\
(0-1)\end{array}$ & $\begin{array}{c}3 \\
(2-4)\end{array}$ & $\begin{array}{c}6 \\
(4-8)\end{array}$ \\
\hline Urban residence $†$ & $\begin{array}{c}3264235 \\
\quad(89.6)\end{array}$ & $\begin{array}{c}3264235 \\
(100.0)\end{array}$ & $\begin{array}{c}0 \\
(0.0)\end{array}$ & $\begin{array}{c}1428028 \\
(89.9)\end{array}$ & $\begin{array}{c}1371563 \\
(90.2)\end{array}$ & $\begin{array}{c}464644 \\
(87.4)\end{array}$ & $\begin{array}{c}1652302 \\
(89.6)\end{array}$ & $\begin{array}{c}1611933 \\
(89.7)\end{array}$ & $\begin{array}{c}2330393 \\
\quad(90.2)\end{array}$ & $\begin{array}{c}933842 \\
(88.3)\end{array}$ & $\begin{array}{l}38674 \\
(88.0)\end{array}$ \\
\hline $\begin{array}{l}\text { Saw primary care } \\
\text { physician in office } \\
\text { setting }\end{array}$ & $\begin{array}{c}2620547 \\
(68.2)\end{array}$ & $\begin{array}{c}2354013 \\
\quad(72.1)\end{array}$ & $\begin{array}{c}264572 \\
(70.2)\end{array}$ & $\begin{array}{c}1032584 \\
\quad(62.0)\end{array}$ & $\begin{array}{c}1136067 \\
(70.2)\end{array}$ & $\begin{array}{c}451896 \\
(80.9)\end{array}$ & $\begin{array}{l}1199365 \\
\quad(61.2)\end{array}$ & $\begin{array}{c}1421182 \\
\quad(75.6)\end{array}$ & $\begin{array}{c}1684849 \\
(60.7)\end{array}$ & $\begin{array}{c}935698 \\
(87.8)\end{array}$ & $\begin{array}{l}33037 \\
(74.7)\end{array}$ \\
\hline $\begin{array}{l}\text { Saw salaried } \\
\text { specialist as } \\
\text { outpatient }\end{array}$ & $\begin{array}{c}173541 \\
(4.5)\end{array}$ & $\begin{array}{c}158633 \\
(4.9)\end{array}$ & $\begin{array}{c}14878 \\
(3.9)\end{array}$ & $\begin{array}{c}46302 \\
(2.8)\end{array}$ & $\begin{array}{c}77135 \\
(4.8)\end{array}$ & $\begin{array}{c}50104 \\
(9.0)\end{array}$ & $\begin{array}{c}75608 \\
(3.9)\end{array}$ & $\begin{array}{l}97933 \\
(5.2)\end{array}$ & $\begin{array}{l}71407 \\
(2.6)\end{array}$ & $\begin{array}{c}102134 \\
(9.6)\end{array}$ & $\begin{array}{l}5447 \\
(12.3)\end{array}$ \\
\hline $\begin{array}{l}\text { Saw FFS specialist } \\
\text { as outpatient }\end{array}$ & $\begin{array}{c}1221966 \\
(31.8)\end{array}$ & $\begin{array}{c}1108923 \\
(34.0)\end{array}$ & $\begin{array}{c}112086 \\
(29.7)\end{array}$ & $\begin{array}{c}371194 \\
(22.3)\end{array}$ & $\begin{array}{c}546251 \\
(33.8)\end{array}$ & $\begin{array}{c}304521 \\
(54.5)\end{array}$ & $\begin{array}{c}534034 \\
(27.2)\end{array}$ & $\begin{array}{c}687932 \\
(36.6)\end{array}$ & $\begin{array}{c}655537 \\
(23.6)\end{array}$ & $\begin{array}{c}566429 \\
(53.2)\end{array}$ & $\begin{array}{c}20704 \\
(46.8)\end{array}$ \\
\hline $\begin{array}{l}\text { Saw any specialist } \\
\text { as outpatient }\end{array}$ & $\begin{array}{c}1273175 \\
\quad(33.2)\end{array}$ & $\begin{array}{c}1154894 \\
(35.4)\end{array}$ & $\begin{array}{c}117310 \\
(31.1)\end{array}$ & $\begin{array}{c}389028 \\
(23.4)\end{array}$ & $\begin{array}{c}570256 \\
(35.3)\end{array}$ & $\begin{array}{c}313891 \\
(56.2)\end{array}$ & $\begin{array}{c}557575 \\
(28.4)\end{array}$ & $\begin{array}{c}715600 \\
(38.1)\end{array}$ & $\begin{array}{c}683110 \\
(24.6)\end{array}$ & $\begin{array}{c}590065 \\
(55.4)\end{array}$ & $\begin{array}{c}22082 \\
(50.0)\end{array}$ \\
\hline $\begin{array}{l}\text { Had at least } \\
1 \text { medication } \\
\text { dispensation }\end{array}$ & $\begin{array}{c}2313406 \\
(60.2)\end{array}$ & $\begin{array}{c}2058693 \\
(63.1)\end{array}$ & $\begin{array}{c}252755 \\
(67.0)\end{array}$ & $\begin{array}{c}820186 \\
(49.3)\end{array}$ & $\begin{array}{c}1047437 \\
(64.8)\end{array}$ & $\begin{array}{c}445783 \\
(79.8)\end{array}$ & $\begin{array}{c}1047875 \\
(53.4)\end{array}$ & $\begin{array}{c}1265531 \\
(67.3)\end{array}$ & $\begin{array}{c}1388384 \\
(50.0)\end{array}$ & $\begin{array}{c}925022 \\
(86.8)\end{array}$ & $\begin{array}{l}36653 \\
(82.9)\end{array}$ \\
\hline $\begin{array}{l}\text { Had at least } \\
1 \text { emergency } \\
\text { department visit }\end{array}$ & $\begin{array}{c}735973 \\
(19.2)\end{array}$ & $\begin{array}{c}613342 \\
(18.8)\end{array}$ & $\begin{array}{l}121334 \\
(32.2)\end{array}$ & $\begin{array}{c}313954 \\
(18.9)\end{array}$ & $\begin{array}{c}278553 \\
(17.2)\end{array}$ & $\begin{array}{c}143466 \\
(25.7)\end{array}$ & $\begin{array}{c}353512 \\
(18.0)\end{array}$ & $\begin{array}{c}382461 \\
(20.4)\end{array}$ & $\begin{array}{c}432441 \\
(15.6)\end{array}$ & $\begin{array}{c}303532 \\
(28.5)\end{array}$ & $\begin{array}{l}21678 \\
(49.0)\end{array}$ \\
\hline $\begin{array}{l}\text { Had at least } \\
1 \text { hospital admission }\end{array}$ & $\begin{array}{c}215148 \\
(5.6)\end{array}$ & $\begin{array}{c}184571 \\
(5.7)\end{array}$ & $\begin{array}{c}30288 \\
(8.0)\end{array}$ & $\begin{array}{c}83562 \\
(5.0)\end{array}$ & $\begin{array}{c}66848 \\
(4.1)\end{array}$ & $\begin{array}{l}64738 \\
(11.6)\end{array}$ & $\begin{array}{c}80152 \\
(4.1)\end{array}$ & $\begin{array}{c}134996 \\
(7.2)\end{array}$ & $\begin{array}{l}101565 \\
(3.7)\end{array}$ & $\begin{array}{c}113583 \\
(10.7)\end{array}$ & $\begin{array}{l}15744 \\
(35.6)\end{array}$ \\
\hline $\begin{array}{l}\text { Admitted to } \\
\text { academic centre }\end{array}$ & $\begin{array}{c}47642 \\
(1.2)\end{array}$ & $\begin{array}{c}43213 \\
(1.3)\end{array}$ & $\begin{array}{c}4354 \\
(1.2)\end{array}$ & $\begin{array}{c}13120 \\
(0.8)\end{array}$ & $\begin{array}{c}18020 \\
(1.1)\end{array}$ & $\begin{array}{c}16502 \\
(3.0)\end{array}$ & $\begin{array}{c}22828 \\
(1.2)\end{array}$ & $\begin{array}{c}24814 \\
(1.3)\end{array}$ & $\begin{array}{c}17975 \\
(0.6)\end{array}$ & $\begin{array}{c}29667 \\
(2.8)\end{array}$ & $\begin{array}{l}4248 \\
(9.6)\end{array}$ \\
\hline $\begin{array}{l}\text { Pharmacist- } \\
\text { delivered patient } \\
\text { counselling or } \\
\text { prescription } \\
\text { modification }\end{array}$ & $\begin{array}{c}169243 \\
(3.7)\end{array}$ & $\begin{array}{c}127315 \\
(3.5)\end{array}$ & $\begin{array}{c}41928 \\
(4.4)\end{array}$ & $\begin{array}{c}36917 \\
(2.4)\end{array}$ & $\begin{array}{c}65716 \\
(4.4)\end{array}$ & $\begin{array}{c}48645 \\
(8.5)\end{array}$ & $\begin{array}{c}73820 \\
(3.2)\end{array}$ & $\begin{array}{c}95423 \\
(4.2)\end{array}$ & $\begin{array}{c}167894 \\
(3.7)\end{array}$ & $\begin{array}{c}1349 \\
(13.7)\end{array}$ & NA \\
\hline
\end{tabular}

was $84.4 \%$ (standard error $0.4 \%$ ) (data not shown). Overall, $3.2 \%$ of adults reported they had unmet health care needs in the prior year; the proportion was significantly higher for younger adults (age 18-40), women and those with at least 1 ACSC (all $p<0.001)$ (Appendix 2).

\section{Alberta Health Link teletriage advice}

Of the 470207 adults who called Health Link in 2018 (12.2\% of all adults and $3.5 \%$ of those with ACSCs), $269429(57.3 \%)$ did so to ask a question about their own symptoms, and 157049 (33.4\%) were advised to proceed to an emergency department or medical provider within 4 hours (Appendix 2). Proportions were similar across the subgroups examined (Appendix 2), although patients with at least 1 ACSC were more likely to be advised to present to an emergency department or physician within 4 hours than those without ACSCs (45.2\% v. 32.3\%, $p=0.001)$.

Figure 3 summarizes the ecology of care for communitydwelling adults in Alberta in 2016/17.

\section{Interpretation}

Between 2002/03 and 2016/17, the community-dwelling adult population of Alberta became older (median age increased by $2 \mathrm{yr}$ ), and the proportion with at least $1 \mathrm{ACSC}$ increased from $21 \%$ to $28 \%$. Despite an absolute decrease of $2.6 \%$ in the proportion of community-dwelling people seen by a primary care physician, an absolute decrease of $1.4 \%$ in the proportion who presented to an emergency department, an absolute increase of $1.3 \%$ in the proportion 


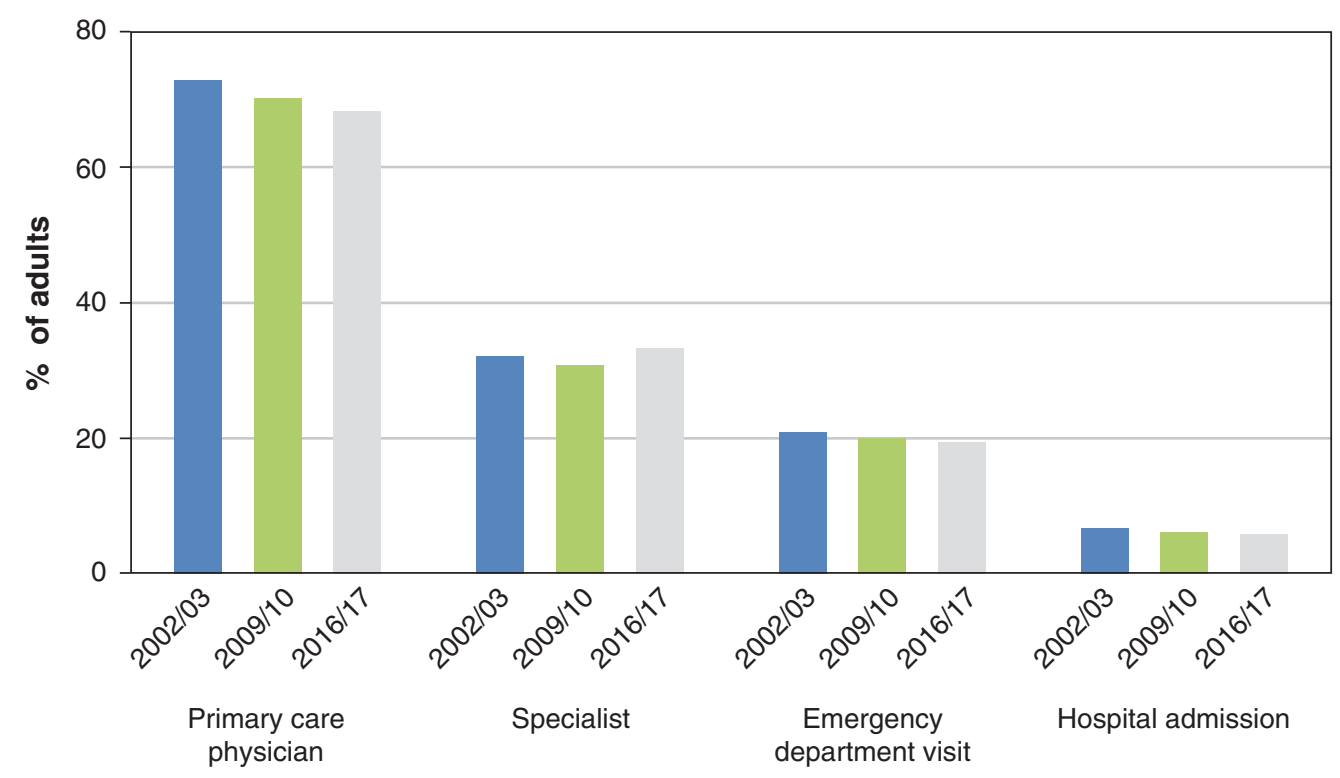

Figure 1: Proportions of community-dwelling adult Albertans who saw a primary care physician, saw a specialist, visited an emergency department and were admitted to hospital in 2002/03, 2009/10 and 2006/17.

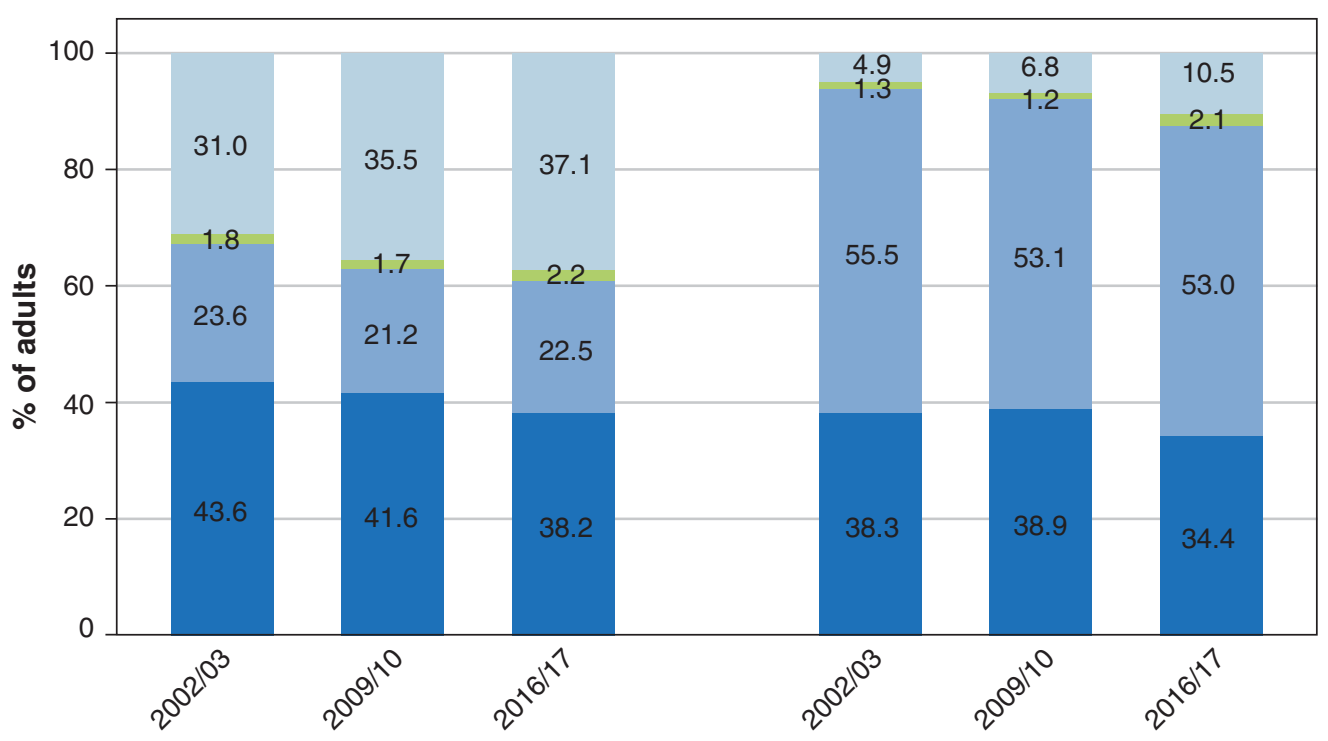

No ACSC

ACSC

\begin{tabular}{|l|l|}
\hline PCP only & PCP and specialist \\
Specialist only & None \\
\hline
\end{tabular}

Figure 2: Proportions of community-dwelling adults with and without at least 1 ambulatory-care-sensitive condition (ACSC) who visited an outpatient clinic in 2002/03, 2009/10 and 2006/17. Note: PCP = primary care physician. 


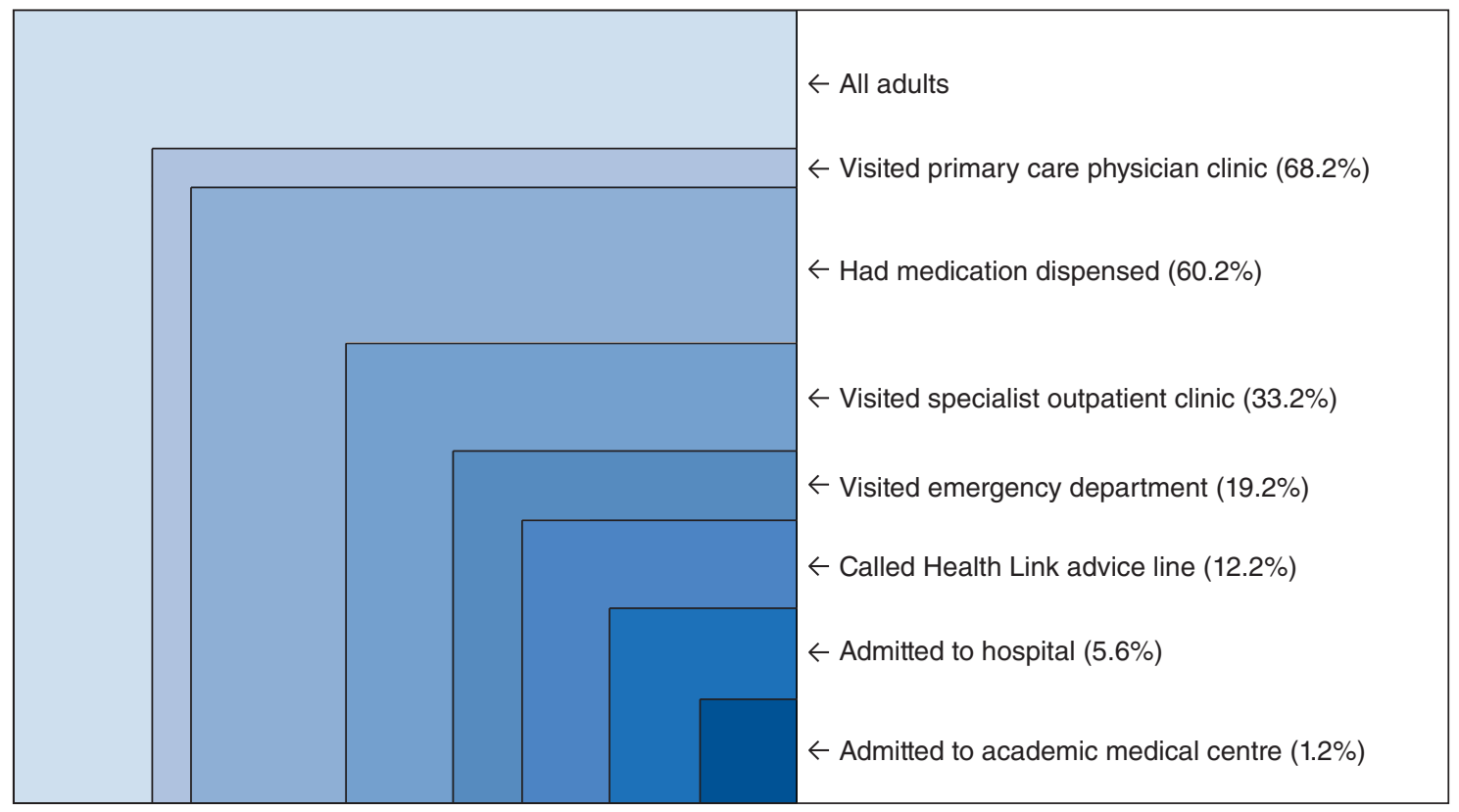

Figure 3: The ecology of medical care for community-dwelling adults in Alberta, 2016/17. The sets of patients are not necessarily nested within each other.

who saw a specialist (mostly owing to increases in the proportion seeing salaried specialists) and an absolute increase of $5.3 \%$ between $2009 / 10$ and 2016/17 in the proportion who received at least 1 medication dispensation, the proportions admitted to hospital or an academic hospital were relatively stable over the study period $(6 \%$ and $1 \%$, respectively). Despite introduction of specific fee payments for counselling from pharmacists or having outpatient prescriptions modified, we found that few Albertans $(<1$ in 27 overall and $<1$ in 7 with ACSCs) received these services. However, 1 in 8 adults (but only 1 in 29 of those with ACSCs) accessed the Alberta Health Link teletriage line for health advice.

In contrast to data from US studies, ${ }^{2,3}$ communitydwelling adults in Alberta were more than twice as likely to see a primary care physician than a specialist. Although it is not possible to compare absolute outpatient visit rates in the United States and Canada from prior published studies, 2,3 according to the Medical Expenditure Panel Survey website (https://meps.ahrq.gov/mepstrends/hc_use/), the proportion of community-dwelling US adults who had an outpatient physician visit was lower than that in Alberta during all 3 years studied and also declined between 2003 and 2016 (from $68 \%$ to $65 \%$ ). Of note, at the time of writing, 2016 was the most recent year of data available on the Medical Expenditure Panel Survey website. Although survey participants in the US were less likely to present to an emergency department in a year (about 13\%) than Alber- tans, they were slightly more likely to be admitted to hospital (about 8\%-9\% per year), and, as in Alberta, these proportions did not change appreciably between 2003 and 2016. Thus, although the trends since 2003 were similar in Alberta and the US, the ecology of medical care differed in that adults in Alberta were more likely to see a primary care physician or present to an emergency department than their US counterparts but were less likely to be admitted to hospital.

In the current study, patients with ACSCs were far more likely than those without ACSCs to receive outpatient care from their primary care physician or a specialist, and to have at least 1 medication dispensed; they were also more likely to have an emergency department visit or be admitted to hospital (with similar relative proportions in all years studied). This finding raises questions about whether emergency department visits or admissions for ACSCs truly are preventable with outpatient care. Our results are consistent with previous work showing that ACSC-related emergency department visits and hospital admissions are not sensitive to the frequency of outpatient care received. ${ }^{17-22}$

About $1 \%$ of adults in Alberta were long-term care residents in the years we studied. These people had a median of 6 comorbidities, and, although the proportion who saw a specialist in an outpatient setting increased over the study period and approached $50 \%$ in $2016 / 17$, they were still more than twice as likely as community-dwelling adults to present to an emergency department, 6 times more likely to be admitted to 
a hospital and 10 times more likely to be admitted to an academic tertiary care hospital. Without information on the goals of care for these long-term care residents, we cannot comment on the appropriateness of this pattern of use of health care services, but it appears to be an area where future policy changes might be targeted fruitfully.

The stability of patterns of health care delivery between 2002/03 and 2016/17 in Alberta is consistent with prior publications in other settings showing little change in the ecology of care between the 1930s and the early 2000s. ${ }^{1-3}$ Thus, it appears that inertia in health care affects more than just the therapy decision-making of clinicians and patients. ${ }^{23,24}$

\section{Limitations}

Although we were able to examine all interactions with physicians, pharmacists, emergency departments and hospitals for virtually all adults in an entire Canadian province, there are some limitations to our study. First, and most important, our data did not permit us to examine the efficiency or quality of care provided to adult Albertans. However, a recent analysis of 195 countries in the Global Burden of Disease Study showed that Canada was in the top $10 \%$ on the Healthcare Access and Quality Index. ${ }^{25}$ Second, we relied on administrative data to define ACSCs and do not have information on clinical assessment or biomarkers that would delineate the severity of each condition. However, we used validated International Statistical Classification of Diseases and Related Health Problems, 10th Revision case definition algorithms to assign diagnoses. ${ }^{14}$ Third, we were unable to examine visits to nonphysician health care providers, such as public health nurses, dentists and chiropractors, or other aspects of use of health care services, such as laboratory and diagnostic imaging visits. Finally, as with any observational study, the impact of unmeasured confounders is unknown and limits our ability to make definitive statements on causation. Thus, in this study, we can only describe care patterns in different years rather than attribute success or failure to each policy change. Indeed, as we had access to only 3 years' worth of data, we could only describe patterns of care in each year rather than do more elaborate analyses such as interrupted time-series or multivariable regression analyses.

\section{Conclusion}

We have shown that the ecology of medical care changed little in Alberta between 2002/03 and 2016/17 despite the implementation of 5 province-wide system changes explicitly designed to alter care patterns by increasing the use of pharmacists and specially trained teletriage nurses. and to influence the delivery of primary care and specialist services. Rather than searching for a policy "magic bullet," health care planners may be better served by focusing on upscaling and implementing interventions already tested and proven to be effective in pilot studies. However, as not all good ideas have the anticipated effects, any policy implementation should be accompanied by robust evaluation plans.

\section{References}

1. White KL, Williams TF, Greenberg BG. The ecology of medical care. N Engl 7 Med 1961;265:885-92.

2. Green LA, Fryer GE Jr, Yawn BP, et al. The ecology of medical care revisited. N Engl f Med 2001;344:2021-5.

3. Johansen ME, Kircher SM, Huerta TR. Reexamining the ecology of medical care. N Engl 7 Med 2016;374:495-6.

4. Sturmberg JP, McDonnell GM. How modelling could contribute to reforming primary care - tweaking "the ecology of medical care" in Australia. AIMS Med Sci 2016;3:298-311.

5. Fryer GE Jr, Green LA, Dovey SM, et al. Variation in the ecology of medical care. Ann Fam Med 2003;1:81-9.

6. Tonelli M, Wiebe N, Manns BJ, et al. Comparison of the complexity of patients seen by different medical specialists in a universal health care system. FAMA Netw Open 2018;1:e184852.

7. Pilla SJ, Segal JB, Maruthur NM. Primary care provides the majority of outpatient care for patients with diabetes in the US: NAMCS 2009-2015. 7 Gen Intern Med 2019;34:1089-91.

8. McAlister FA, Bakal JA, Green L, et al. The effect of provider affiliation with a primary care network on emergency department visits and hospital admissions. CMA7 2018;190:E276-84.

9. Bichel A, Bacchus M, Meddings J, et al. Academic alternate relationship plans for internal medicine: a lever for health care transformation. Open Med 2011;5: e28-32.

10. Manns BJ, Wasylak T. Clinical networks: enablers of health system change. CMA7 2019;191:E1299-305.

11. De Coster C, Quan H, Elford R, et al. Follow-through after calling a nurse telephone advice line: a population-based study. Fam Pract 2010;27:271-8.

12. Disparities in primary health care experiences among Canadians with ambulatory care sensitive conditions. Ottawa: Canadian Institute for Health Information; 2012. Available: https://secure.cihi.ca/free_products/PHC_Experiences_ AiB2012_E.pdf (accessed 2020 Mar. 2).

13. Canadian Community Health Survey. Available: https://www.canada.ca/en/ health-canada/services/food-nutrition/food-nutrition-surveillance/health -nutrition-surveys/canadian-community-health-survey-cchs.html (accessed 2020 Mar. 2)

14. Tonelli M, Wiebe N, Fortin M, et al.; Alberta Kidney Disease Network. Methods for identifying 30 chronic conditions: application to administrative data. BMC Med Inform Decis Mak 2015;15:31.

15. Ye M, Vena JE, Johnson JA, et al. Validation of drug prescription records for senior patients in Alberta's Tomorrow Project: assessing agreement between two population-level administrative pharmaceutical databases in Alberta, Canada. Pharmacoepidemiol Drug Saf 2019;28:1417-21.

16. Cuzick J. A Wilcoxon-type test for trend. Stat Med 1985;4:87-90

17. Weinberger M, Oddone EZ, Henderson WG. Does increased access to primary care reduce hospital readmissions? Veterans Affairs Cooperative Study Group on Primary Care and Hospital Readmission. N Engl 7 Med 1996;334: $1441-7$.

18. Rosano A, Loha CA, Falvo R, et al. The relationship between avoidable hospitalization and accessibility to primary care: a systematic review. Eur $\mathcal{F}$ Public Health 2013;23:356-60.

19. Walker RL, Chen G, McAlister FA, et al.; Hypertension Outcome and Surveillance Team. Relationship between primary care physician visits and hospital/emergency use for uncomplicated hypertension, an ambulatory caresensitive condition. Can 7 Cardiol 2014:30:1640-8.

20. Solberg LI. Preventable hospital admissions: Are they? Fam Pract 2015;32: 245-6.

21. Vuik SI, Fontana G, Mayer E, et al. Do hospitalisations for ambulatory care sensitive conditions reflect low access to primary care? An observational cohort study of primary care usage prior to hospitalization. BMF Open 2017;7:e15704.

22. Paul MC, Dik JH, Hoekstra T, et al. Admissions for ambulatory care sensitive conditions: a national observational study in the general and COPD population. Eur F Public Health 2019;29:213-9.

23. Phillips LS, Branch WT, Cook CB, et al. Clinical inertia. Ann Intern Med 2001:135:825-34.

24. Majumdar SR, McAlister FA, Furberg CD. From publication to practice in chronic cardiovascular disease: a long and winding road. 7 Am Coll Cardiol 2004:43:1738-42.

25. GBD 2015 Healthcare Access and Quality Collaborators. Healthcare Access and Quality Index based on mortality from causes amenable to personal health care in 195 countries and territories, 1990-2015: a novel analysis from the Global Burden of Disease Study 2015. Lancet 2017;390:231-66.

Affiliations: Division of General Internal Medicine (McAlister), Alberta SPOR Support Unit Data Platform (McAlister, Lin) and Patient Health Outcomes Research and Clinical Effectiveness Unit (McAlister, Lin), University of Alberta, Edmonton, Alta.; Departments of Medicine (Tonelli, Svenson) and Community Health Sciences (Tonelli, Svenson), Cumming School of Medicine, University of Calgary, Calgary, Alta.; Division of Nephrology (Wiebe), Department of Medicine, University of 
Alberta; Alberta Health (Svenson); Division of Preventive Medicine (Svenson) and School of Public Health (Svenson), University of Alberta, Edmonton, Alta.; Data Integration Measurement and Reporting (Dean), Alberta Health Services, Calgary, Alta.

Contributors: Finlay McAlister conceived and designed the study, interpreted the data and drafted the manuscript. Natasha Wiebe and Meng Lin analyzed the data. All of the authors revised the manuscript critically for important intellectual content, approved the final version to be published and agreed to be accountable for all aspects of the work.

Funding: Funding was provided by a team grant to the Interdisciplinary Chronic Disease Collaboration from Alberta Innovates and a Leaders Opportunity Fund grant from the Canada Foundation for Innovation. Finlay McAlister is supported by the Alberta Health Services Chair in Cardiovascular Outcomes Research. Marcello Tonelli is supported by the David Freeze Chair in Health Services Research at the University of Calgary.

Acknowledgement: The authors thank Dr. Leiah Luoma for her assistance with generating Figure 3.
Data-sharing statement: We are not able to make our data set available to other researchers owing to our contractual arrangements with the provincial health ministry (Alberta Health), who is the data custodian. Researchers may make requests to obtain a similar data set at https:// albertainnovates.ca/our-health-innovation-focus/the-alberta-spor-support -unit/.

Disclaimer: This study is based in part by data provided by Alberta Health and Alberta Health Services. The interpretation and conclusions contained herein are those of the researchers and do not represent the views of the Government of Alberta or Alberta Health Services. Neither the Government of Alberta nor Alberta Health or Alberta Health Services express any opinion in relation to this study. The sponsors had no role in the design and conduct of the study; collection, management, analysis and interpretation of the data; preparation, review or approval of the manuscript; or in the decision to submit the manuscript for publication.

Supplemental information: For reviewer comments and the original submission of this manuscript, please see www.cmajopen.ca/content/8/1/ E169/suppl/DC1. 